\title{
Präinterventionelle Änderung der Gerinnungsmedikation
}

\author{
Jürgen Koscielny Edita Rutkauskaite \\ Institut für Transfusionsmedizin, Universitätsmedizin Berlin, Universitätsklinikum Charité, Campus Charité Mitte, Berlin, Deutschland
}

Schlüsselwörter

Gerinnungsmedikation · Hämorrhagische Diathese · Präoperatives Management

\section{Zusammenfassung}

Hintergrund: Die Differentialdiagnostik und Therapie von perioperativen Blutungen besitzt besonders bei Eingriffen mit hohen Blutverlusten einen hohen Stellenwert, z.B. in der Viszeralchirurgie. Insbesondere die präoperative Erfassung von hämorrhagischen Diathesen und einer gerinnungshemmenden Medikation trägt effektiv zur Minimierung von Komplikationen wie Hämatomen, sekundären Infektionen und Blutverlusten bei. Methode: Pharmakologische Aspekte, bereits vorliegende Konsensus-Empfehlungen und Leitlinien, Erfahrungen sowie Empfehlungen der Hersteller und der Zulassungsbehörden wurden genutzt. Ergebnisse: Bei Elektiveingriffen unter Langzeitantikoagulation ist zur Normalisierung des Blutungsrisikos bei normaler Nieren- und Leberfunktion ein Absetzen der Gerinnungsmedikation vor der Operation ausreichend, ein längerer Abstand ist bei eingeschränkter Nieren- und Leberfunktion sowie bei hohem Lebensalter zu berücksichtigen. Bei Patienten mit positiver standardisierter Blutungsanamnese durch die Einnahme von gerinnungshemmenden Medikamenten sollte auf der Basis einer individuellen Nutzen-Risiko-Abwägung (Individualmedizin) interdisziplinär das perioperative Weiterführen oder Absetzen von Gerinnungshemmern festgelegt werden. Da die Halbwertszeit aber interindividuell unterschiedlich und zum Teil unvorhersehbar sein kann, insbesondere bei Patienten mit verminderter Elimination (z.B. Niereninsuffizienz), kann in Einzelfällen ein geeigneter laboranalytischer Wirkungsnachweis als zusätzliche Entscheidungshilfe die Terminierung der Intervention unterstützen. Aus hämostaseologischer Sicht besteht die Indikation zum bereits präoperativen Management einer hämorrhagischen Diathese. Die Maßnahmen richten sich nach der jeweiligen Defizienz. Schlussfolgerungen: Elektive Eingriffe müssen sorgfältig geplant werden, besonders bei Patienten mit Gerinnungsmedikation oder hämorrhagischer Diathese.

\section{KARGER \\ Fax +497614520714 \\ Information@Karger.com}

www.karger.com (c) 2013 S. Karger GmbH, Freiburg

$1662-6664 / 13 / 0295-0266 \$ 38.00 / 0$

Accessible online at:

www.karger.com/vim
Keywords

Anticoagulant medication - Hemorrhagic diathesis . Preoperative management

\section{Summary}

\section{Preinterventional Changes in Clotting Medication}

Background: The differential diagnosis and treatment of perioperative bleeding has a high value especially during interventions with high blood loss, e.g. in visceral surgery. In particular, the preoperative detection of hemorrhagic diathesis and anticoagulant medication helps to effectively minimize complications such as hematoma, secondary infection, and blood loss. Method: Pharmacological aspects, the current consensus recommendations and guidelines, and the experiences and recommendations of the manufacturers and regulatory authorities were used. Results: During elective invasion under long-term anticoagulation therapy to normalize the bleeding risk under normal renal and hepatic function, discontinuation of the anticoagulant medication before surgery is sufficient; a longer distance must be considered in renal and hepatic impairment and in advanced age. In patients with positive bleeding history by taking anticoagulant drugs, the interdisciplinary perioperative continuation or discontinuation of anticoagulants should be based on an individual benefit-risk assessment (individualized medicine). Since the half-life may vary inter-individually and may be partly unpredictable, especially in patients with decreased elimination (e.g. renal failure), an appropriate analytical-laboratory proof of effect can, in individual cases, support the scheduling of intervention, as an additional decision basis. From the blood coagulation view, the indication for preoperative management of hemorrhagic diathesis and a change in the anticoagulant medication is given. The measures depend on the particular deficiency. Conclusions: Elective surgery must be carefully planned, especially in patients with anticoagulation medication or hemorrhagic diathesis. 


\section{Einleitung}

Perioperative Blutungen sind trotz moderner Operationstechniken und intensiver Überwachung der Patienten ein alltägliches klinisches Problem. Die Differentialdiagnostik und Therapie von perioperativen Blutungen besitzt besonders bei Eingriffen mit hohen Blutverlusten einen sehr hohen Stellenwert, z.B. in der Viszeralchirurgie, Kardiochirurgie und Orthopädie. Dies ist unter anderem eine Ursache für den hohen Verbrauch von mindestens 7,2 Millionen Blutkomponenten (Erythrozytenkonzentrat, Thrombozytenkonzentrat, gefrorenes Frischplasma) in Deutschland pro Jahr [1]. Grundsätzlich kommen für die perioperativen Blutungen entweder chirurgische und/oder hämostaseologische Ursachen infrage. Daher ist es indiziert, $\mathrm{Pa}$ tienten mit einer hämorrhagischen Diathese oder einer gerinnungshemmenden Medikation bereits präoperativ zu erfassen und adäquat vorzubereiten. Insbesondere die präoperative Erfassung von hämorrhagischen Diathesen und einer gerinnungshemmenden Medikation trägt effektiv zur Minimierung von Komplikationen wie Hämatomen, sekundären Infektionen und Blutverlusten bei. Immerhin benötigen etwa 5\% der Patienten nach Stentimplantation oder Patienten mit hämorrhagischer Diathese eine nichtkardiale Operation innerhalb eines Jahres [2]. Daher ist die Verunsicherung in der nichtkardiochirurgischen operativen Medizin groß. Im Folgenden werden die klinische Problematik und Lösungsmöglichkeiten beschrieben.

\section{Risikobewertung}

Das perioperative Blutungsrisiko eines Patienten mit einer hämorrhagischen Diathese oder unter einer gerinnungshem- menden Medikation wird von mehreren Faktoren beeinflusst (Abb. 1):

- Art und «Größe» der Operation (Traumatisierungsgrad);

- Operationsart mit einer Transfusionswahrscheinlichkeit $>10 \%$ (CAVE: jährliche meldepflichtige krankenhauseigene Daten (Richtlinien zur Hämotherapie));

- gerinnungshemmende Medikation und weitere Medikation zur Nieren- und Leberfunktion;

- aktueller klinischer Zustand (internationale Stadien der American Society of Anesthesiology (ASA-Stadien));

- Art der hämorrhagischen Diathese;

- klinische Ausprägung der hämorrhagischen Diathese (CAVE: Blutungsanamnese).

Somit bestimmen die Disposition des Patienten (Art der hämorrhagischen Diathese oder der gerinnungshemmenden Medikation, klinische Ausprägung mit Blutungsanamnese, aktueller klinischer Zustand, Medikation) und die operative Exposition (Art und «Größe» der Operation) das perioperative Blutungsrisiko [3, 4]. Aus klinischer Sicht besteht ein besonders hohes perioperatives Blutungsrisiko für Patienten mit hämorrhagischer Diathese oder unter einer gerinnungshemmenden Medikation, wenn eine oder mehrere der folgenden Bedingungen anzutreffen sind:

- «große» traumatisierende Operationen aus den Bereichen z.B. der Kardiochirurgie, Neurochirurgie, Tumorchirurgie, Orthopädie (Knie, Hüfte, Wirbelsäule) oder Kieferchirurgie (ausgedehnte Eingriffe);

- bei Schleimhauteingriffen wie z.B. im Hals/Nasen/ Ohren(HNO)-Bereich, im Gastrointestinaltrakt und im Urogenitaltrakt sowie im gynäkologischen Bereich, z.B. Sectio caesarea, Hysterektomie;

- Operationsgebiete mit hoher Konzentration an fibrinolyse-
Abb. 1. Präoperativer Algorithmus basierend auf einer standardisierten Blutungsanamnese.

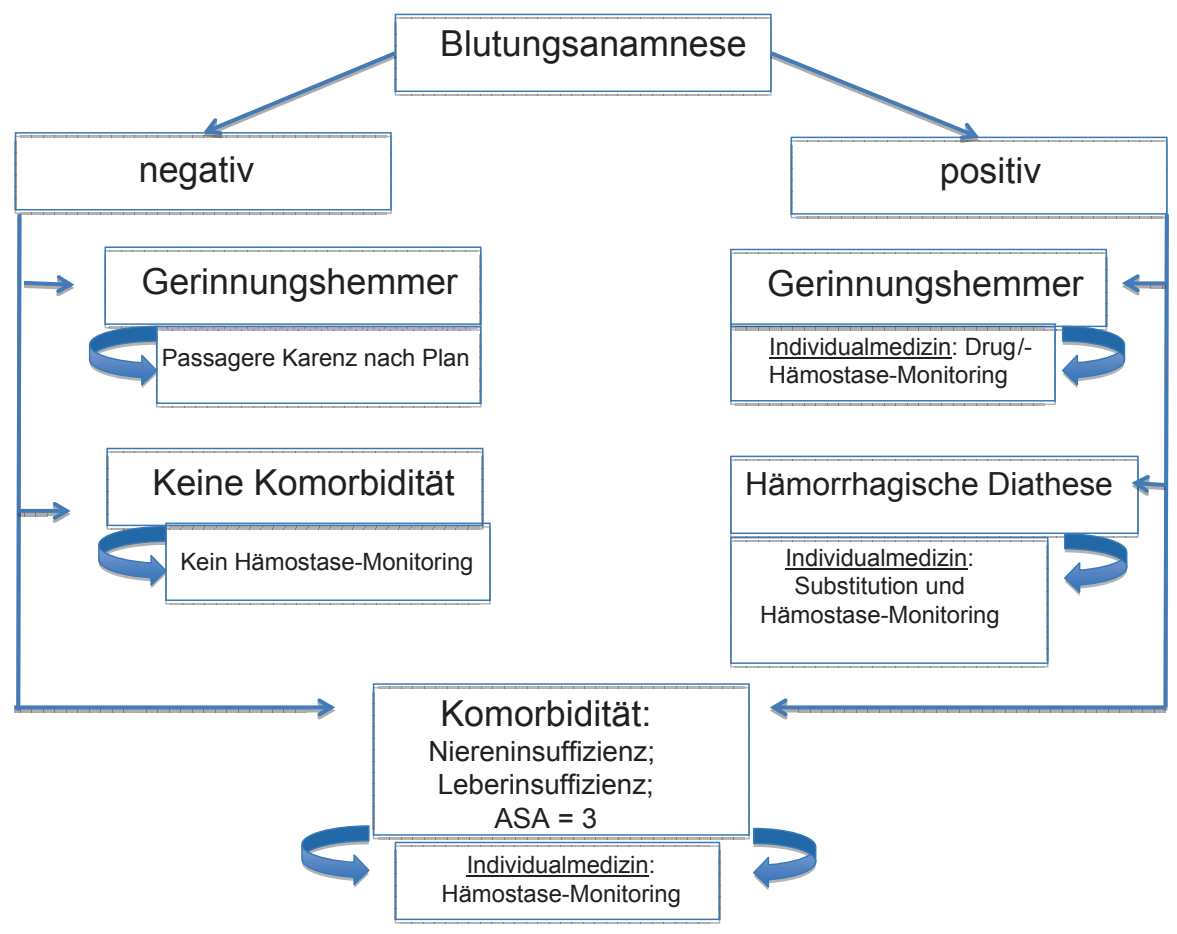


Tab. 1. Blutungsanamnesebogen (grau unterlegt: vom Patienten zu lesen und auszufüllen)

\begin{tabular}{|c|c|c|c|c|c|}
\hline \multicolumn{2}{|r|}{$\begin{array}{l}\text { Bitte Zutreffendes ankreuzen, unterstreichen bzw. } \\
\text { ergänzen: }\end{array}$} & \multirow{2}{*}{$\begin{array}{l}\mathbf{N} \\
\text { Nein } \\
\mathbf{N} \\
\text { Nein }\end{array}$} & \multirow{2}{*}{\begin{tabular}{|l|}
$\mathbf{J}$ \\
$\mathrm{Ja}$ \\
$\mathbf{J}$ \\
$\mathrm{Ja}$ \\
\end{tabular}} & \multirow{2}{*}{$\begin{array}{l}\text { Zusatzfragen und Notizen des Arztes: } \\
\text { Diagnose erfragen }\end{array}$} & \multirow{2}{*}{$\frac{\text { wenn JA }}{2}$} \\
\hline 0 & $\begin{array}{l}\text { Ist bei Ihnen jemals eine Blutgerinnungsstörung oder } \\
\text { Thrombose festgestellt worden? }\end{array}$ & & & & \\
\hline \multicolumn{6}{|c|}{$\begin{array}{l}\text { Beobachten Sie folgende Blutungsarten - auch ohne } \\
\text { erkennbaren Grund? }\end{array}$} \\
\hline $1 \mathrm{a}$ & $\begin{array}{l}\text { Nasenbluten (ohne andere Ursachen wie Schnupfen, } \\
\text { trockene Luft, starkes Naseputzen usw.) }\end{array}$ & $\begin{array}{l}\mathbf{N} \\
\text { Nein }\end{array}$ & $\mathrm{J}$ & $\begin{array}{l}\text { immer schon } \\
\text { nur saisonal } \\
\text { HNO-Befund vorhanden } \\
\text { bei Medikamenteneinnahme } \\
\text { arterielle Hypertonie }\end{array}$ & $\begin{array}{l}2 \\
3 \\
1 \\
4\end{array}$ \\
\hline $1 \mathrm{~b}$ & $\begin{array}{l}\text { Blaue Flecken oder punktförmige Blutungen (auch } \\
\text { am Körperstamm, auch ohne sich anzustoßen) }\end{array}$ & $\begin{array}{l}\mathbf{N} \\
\text { Nein }\end{array}$ & $\mathrm{J}$ & $\begin{array}{l}\text { unfallträchtige Tätigkeiten } \\
\text { immer schon } \\
\text { bei Medikamenteneinnahme }\end{array}$ & $\begin{array}{l}0 \\
2 \\
1\end{array}$ \\
\hline $1 \mathrm{c}$ & $\begin{array}{l}\text { Gelenksblutungen, Blutungen in Weichteilen oder } \\
\text { Muskeln }\end{array}$ & $\begin{array}{l}\mathbf{N} \\
\text { Nein }\end{array}$ & $\mathrm{J}$ & & 2 \\
\hline 2 & $\begin{array}{l}\text { Beobachten Sie bei Schnittwunden und/oder } \\
\text { Schürfwunden ein längeres Nachbluten? }\end{array}$ & $\begin{array}{l}\mathbf{N} \\
\text { Nein }\end{array}$ & $\mathrm{J}$ & $\begin{array}{l}\text { über } 5 \text { Minuten } \\
\text { typische Verletzung, Nassrasur } \\
\text { bei Medikamenteneinnahme }\end{array}$ & $\begin{array}{l}2 \\
2 \\
1\end{array}$ \\
\hline 3 & $\begin{array}{l}\text { Gab es in Ihrer Vorgeschichte längeres / verstärktes } \\
\text { Nachbluten beim Zahnziehen? }\end{array}$ & $\begin{array}{l}\mathbf{N} \\
\text { Nein }\end{array}$ & $\mathrm{J}$ & $\begin{array}{l}\text { über } 5 \text { Minuten } \\
\text { war Nachbehandlung nötig } \\
\text { bei Medikamenteneinnahme }\end{array}$ & $\begin{array}{l}2 \\
2 \\
1\end{array}$ \\
\hline 4 & $\begin{array}{l}\text { Gab es in Ihrer Vorgeschichte eine verstärkte } \\
\text { Blutung während oder nach Operationen? }\end{array}$ & $\begin{array}{l}\mathbf{N} \\
\text { Nein }\end{array}$ & $\mathrm{J}$ & $\begin{array}{l}\text { welche Operation } \\
\text { war die Blutung tatsächlich über der Norm }\end{array}$ & $\begin{array}{l}5 \\
5 \\
2\end{array}$ \\
\hline 5 & Heilen Ihre Wunden schlecht ab? & $\begin{array}{l}\mathbf{N} \\
\text { Nein }\end{array}$ & $\mathrm{J}$ & $\begin{array}{l}\text { lange nässend, klaffend } \\
\text { Vereitern } \\
\text { Kelloidbildung }\end{array}$ & $\begin{array}{l}2 \\
2 \\
2\end{array}$ \\
\hline 6 & $\begin{array}{l}\text { Gab / gibt es in Ihrer Familie (Blutsverwandtschaft) } \\
\text { Fälle von Blutungsneigung? }\end{array}$ & $\begin{array}{l}\mathbf{N} \\
\text { Nein }\end{array}$ & $\begin{array}{l}\mathrm{J} \\
\mathrm{Ja}\end{array}$ & $\begin{array}{l}\text { Verwandtschaftsgrad } \\
\text { Diagnose bekannt }\end{array}$ & 2 \\
\hline $7 \mathrm{~b}$ & 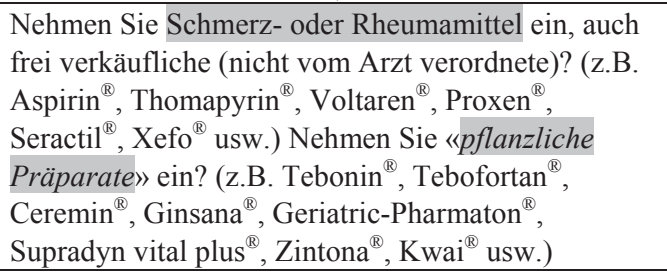 & $\begin{array}{l}\mathbf{N} \\
\text { Nein }\end{array}$ & $\mathrm{J}$ & & \\
\hline 8 & $\begin{array}{l}\text { Zusatzfrage an Patientinnen: Sind Ihre } \\
\text { Monatsblutungen verlängert ( }>7 \text { Tage) und/oder } \\
\text { verstärkt (häufiger Binden-/Tamponwechsel)? }\end{array}$ & Nein & $\mathrm{J}$ & seit Menarche & 2 \\
\hline \multicolumn{6}{|c|}{$\begin{array}{l}\text { Konsequenzen: (0) keine; (1) Medikamentenanamnese; (2) Konsultation: Gerinnungsteam; (3) Konsultation: Facharzt für HNO; (4) } \\
\text { Konsultation: Internist; (5) Befundaushebung; (6) Konsultation: Internist/Chirurg und evtl. Karenz und Bridging. }\end{array}$} \\
\hline
\end{tabular}

aktivierenden Substanzen, z.B. Uterus, Prostata, Malignome, Gehirn, Rückenmark;

- Operationsart mit einer Transfusionswahrscheinlichkeit $>10 \%$;

- schwerwiegende hämorrhagische Diathese (z.B. angeborene oder erworbene plasmatische Hämostasestörungen $<10 \%$ Restaktivität, von-Willebrand-Syndrom (vWS) Typ 3, angeborene Thrombozytenfunktionsstörungen, erworbene Thrombozytenfunktionsstörungen bei fortgeschritte- nen Organerkrankungen oder bei Kombinationen aus gerinnungshemmenden Medikamenten);

- standardisierte Blutungsanamnese [4-6] mit $\geq 3$ positiv beantworteten Fragen, d.h. zahlreiche Blutungssymptome (Tab. 1);

- ASA-Stadium $\geq$ III (schwere Allgemeinerkrankung mit Leistungsminderung und erhöhtem Blutungs- bzw. Thromboserisiko).

Gleichzeitige Operationen, wie etwa eine zusätzliche 
Tab. 2. Präinterventionelle Therapiepausen von gerinnungshemmenden Medikamenten in der plasmatischen Gerinnung (bei Patienten mit normaler Elimination und Organfunktion)

\begin{tabular}{|c|c|c|}
\hline $\begin{array}{l}\text { Gerinnungsmedikamente in der plasmatischen Gerinnung } \\
\text { Substanzen }\end{array}$ & $\begin{array}{l}\text { Therapieende }+ \text { Labortestwert } \\
\text { frühestens vor Operation }\end{array}$ & $\begin{array}{l}\text { Therapiebeginn } \\
\text { nach Operation }\end{array}$ \\
\hline \multicolumn{3}{|l|}{ UFH (Standardheparin) } \\
\hline s.c. oder kontinuierlich i.v. (aPTT < 1,5 Norm) & $3-4 \mathrm{~h}$ & $1 \mathrm{~h}$ \\
\hline Therapeutische Dosis & aPTT oder ACT im Referenzbereich (>3 h) & 6-12 h (i.v.) \\
\hline \multicolumn{3}{|l|}{ NMH } \\
\hline Prophylaktische Dosis & $12 \mathrm{~h}$ & $2 \mathrm{~h}$ \\
\hline Therapeutische Dosis & $24 \mathrm{~h}+$ anti-Xa-Aktivität unter Nachweisgrenze & $2 \mathrm{~h}$ \\
\hline \multicolumn{3}{|l|}{ Direkte Thrombininhibitoren } \\
\hline Desirudin, Lepirudin & $4,5 \mathrm{~h}+\mathrm{aPTT}$ im Referenzbereich & $4 \mathrm{~h}$ \\
\hline Argatroban & $2 \mathrm{~h}+\mathrm{aPTT}$ im Referenzbereich & $4 \mathrm{~h}$ \\
\hline Bivalirudin & $1 \mathrm{~h}+\mathrm{aPTT}$ im Referenzbereich & $4 \mathrm{~h}$ \\
\hline Dabigatran & $24 \mathrm{~h}$ & $4 \mathrm{~h}$ \\
\hline \multicolumn{3}{|l|}{ Synthetisches Pentasaccharid } \\
\hline Fondaparinux ( $\leq 2,5 \mathrm{mg} / \mathrm{Tag})$ & $36 \mathrm{~h}$ & $6 \mathrm{~h}$ \\
\hline \multicolumn{3}{|l|}{ Direkte Faktor-Xa-Inhibitoren } \\
\hline Rivaroxaban & $24 \mathrm{~h}$ & $3 \mathrm{~h}$ \\
\hline Apixaban & $20-30 \mathrm{~h}$ & $4-6 \mathrm{~h}$ \\
\hline Edoxaban & $20-30 \mathrm{~h}$ & $4-6 h$ \\
\hline Heparinoide (s.c.) & Anti-Xa-Aktivität im Referenzbereich (etwa 2 Tage) & $2 \mathrm{~h}$ \\
\hline Vitamin-K-Antagonisten, INR & $<1,4$ (etwa 2 Tage) & sofort \\
\hline Rekombinantes aktiviertes Protein C & $2 \mathrm{~h}$ & $4 \mathrm{~h}$ \\
\hline
\end{tabular}

UFH = Unfraktioniertes Heparin; NMH = niedermolekulares Heparin; aPTT = aktivierte partielle Thromboplastinzeit; ACT = aktivierte Koagulationszeit; INR = International Normalized Ratio.

Zahnsanierung, sollten bei elektiven operativen Eingriffen an Patienten mit schwerer hämorrhagischer Diathese oder volltherapeutischer Antikoagulation wegen der erhöhten Infektionsgefahr vermieden werden. Obwohl blutstillende Medikamente eingespart werden könnten, ist die erhöhte Infektionsgefahr als schwerwiegender Nachteil einzuschätzen. Bei Hemmkörpern gegen Gerinnungsfaktoren, eine besonders schwere Form einer hämorrhagischen Diathese, sollte die Indikation zu einem operativen Eingriff stets sehr kritisch abgewogen werden. Dies gilt insbesondere für elektive Eingriffe. Notfalleingriffe sollten jedoch nicht wegen der sehr großen Blutungsgefährdung verzögert werden.

\section{Präoperative Führung}

\section{Patienten mit gerinnungshemmender Therapie in der plasmatischen Gerinnung}

Aufgrund der zahlreichen gerinnungshemmenden Medikamente werden die wichtigsten Medikamentengruppen einzeln besprochen (Tab. 2). Die Therapiepausezeit vor der Intervention orientiert sich an der Einhaltung der zweifachen Halbwertszeit der jeweiligen Substanz. Zu diesem Zeitpunkt ist $<25 \%$ der pharmakodynamischen Wirkung zu erwarten [7, 8]. Das Blutungsrisiko kann reduziert werden, wenn die blutungsriskante Intervention (Operation, Punktion, Katheter- entfernung) im pharmakokinetischen Talspiegel des gerinnungshemmenden Medikamentes durchgeführt wird. Die Angabe der postoperativen Therapiepausezeiten bis zur nächsten Dosis des Gerinnungsmedikaments ist in der bisherigen Literatur häufig willkürlich festgesetzt. Bei einer Intervention mit Blutungskomplikationen verlängern sich diese Zeitintervalle.

Bei dringlicher, aber Nichtnotfall-Indikation sollten wie vor Elektivoperationen folgende Abwägungen zur Terminierung des OP-Zeitpunktes an erster Stelle stehen:

- Wann war die letzte Einnahme der Antikoagulanzien?

- Wie ist die Nierenfunktion bzw. Leberfunktion?

- Besteht durch den operativen Eingriff ein zusätzlich erhöhtes, perioperatives Blutungsrisiko?

\section{Standardheparin (unfraktioniertes Heparin)}

Bei prophylaktischer Dosierung ist bei elektiven Eingriffen die Therapiepausezeit von mindestens $3 \mathrm{~h}$ einzuhalten. Von der European Society of Anesthesiology (ESA) wird eine Pausezeit von 4-6 h empfohlen [8]. In der Traumatologie wird oftmals zur Thromboseprophylaxe initial unfraktioniertes Heparin (UFH) verabreicht (5000 IE bei Aufnahme) und mit postoperativer Gabe von niedermolekularem Heparin (NMH) kombiniert. $1 \mathrm{~h}$ nach Punktion/Katheterentfernung kann die nächste prophylaktische Heparindosis folgen (1-2 h nach blutiger Punktion) bzw. 4-6 h nach großen Operationen. 
Tab. 3. Algorithmus zur Unterbrechung der Dabigatranetexilat-Therapie vor elektiven chirurgischen Eingriffen bei Patienten mit normalem oder hohem Blutungsrisiko in Abhängigkeit von der Nierenfunktion

\begin{tabular}{|c|c|c|c|}
\hline \multirow[t]{2}{*}{ Nierenfunktion, $\mathrm{CrCl} / \mathrm{min}$} & \multirow[t]{2}{*}{ Geschätzte Halbwertszeit, h } & \multicolumn{2}{|c|}{ Zeit nach der letzten Dosis von Dabigatranetexilat vor der OP } \\
\hline & & normales Blutungsrisiko & hohes Blutungsrisiko oder große OP \\
\hline$>80$ & $\sim 13$ & $24 \mathrm{~h}$ & 2 Tage \\
\hline$>50$ bis $\leq 80$ & $\sim 15$ & 1-2 Tage & 2-3 Tage \\
\hline$>30$ bis $\leq 50$ & $\sim 18$ & 2-3 Tage $(>48 \mathrm{~h})$ & 4 Tage \\
\hline
\end{tabular}

Bei therapeutischer Dosierung ist der laborchemische Nachweis der Normalisierung der aktivierten partiellen Thromboplastinzeit (aPTT) oder der aktivierten Koagulationszeit (ACT) vor der Intervention empfohlen. Eine Vollheparinisierung z.B. in der Herzchirurgie sollte erst nach 6-12 h erfolgen (z.B. Katheteranlage am Vortag der OP).

\section{Niedermolekulare Heparine}

Bei prophylaktischer Dosierung ist die Therapiepausezeit von $12 \mathrm{~h}$ (Tab. 1) einzuhalten. Aufgrund der langen Pausezeit von $24 \mathrm{~h}$ bei therapeutischer Dosierung (Tab. 1) wird empfohlen: Der Anti-Faktor-Xa-Spiegel nach therapeutischer Dosierung sollte unter der Grenze der Nachweisbarkeit liegen. Ein Bridging mit UFH ist möglich.

Etwa $4 \mathrm{~h}$ nach Punktion/Katheterentfernung bzw. $6 \mathrm{~h}$ nach großen Operationen kann die nächste Dosis von NMH folgen.

\section{Pentasaccharid, z.B. Fondaparinux}

Die Thromboseprophylaxe wird üblicherweise erst $6 \mathrm{~h}$ nach der Operation begonnen. Präoperativ ist mindestens eine Karenz von $36 \mathrm{~h}$ einzuhalten. Die lange Halbwertszeit bedingt lange Therapiepausezeiten (Tab. 1), die aber nicht mit einem Effektivitätsverlust der Thromboseprophylaxe einhergehen [9]. Die Antithrombin-vermittelte selektive Hemmung von Faktor Xa kann weder durch aPTT, QuickWert (Thromboplastinzeit (TPZ))/International Normalized Ratio (INR) noch durch die Blutungszeit quantifiziert werden.

\section{Direkte Thrombininhibitoren}

Bei der Indikation dieser Substanzen in der Therapie einer Heparin-induzierten Thrombozytopenie (HIT)-II mit oder ohne manifeste Thrombosen ist im Allgemeinen eine Therapieunterbrechung aufgrund des hohen Thromboserisikos kontraindiziert. Die Indikation für Regionalanästhesien wird also bei Patienten mit HIT selten gegeben sein. Beim Einsatz der Substanzen zur Thromboseprophylaxe (z.B. bei Patienten mit HIT in der Anamnese) ist Folgendes zu beachten: Unter Hirudinen werden häufig Blutungen beobachtet. Zusätzlich zur Therapiepausezeit von 4,5 h wird der laborchemische Nachweis der Normalisierung von aPTT (unter Verwendung von Actin FS ${ }^{\circledR}$ bzw. Neothromtin ${ }^{\circledR}$ ) oder die Ecarin Clotting Time (ECT) empfohlen. Von der ESA wird eine Pausezeit von mehr als $8-10$ h bei Niereninsuffizienz empfohlen [8]. Aufgrund der kurzen Halbwertszeiten von Bivalirudin sind geringe Therapiepausezeiten empfohlen. Für Argatroban ist eine Therapiepausezeit von $2 \mathrm{~h}$ vor Intervention und von $4 \mathrm{~h}$ nach Intervention empfohlen. Eine Leberinsuffizienz bei Argatroban und eine Niereninsuffizienz bei Bivalirudin verlängern diese Zeiten erheblich, sodass ein individuelles Monitoring zu erfolgen hat.

\section{Dabigatran (Pradaxa ${ }^{\circledR}$ )}

Der Hersteller beschreibt Blockadekatheterverfahren als kontraindiziert; es gibt hierzu keine Studiendaten. Abgeleitet von den pharmakologischen Kennzahlen kann bei prophylaktischer Dosis eine Therapiepause von 26 h vor Intervention und von $4 \mathrm{~h}$ nach Intervention [10] abgeleitet werden. Von der ESA wird eine Pausezeit von 6 h empfohlen [8]. Bei Patienten mit Niereninsuffizienz kann die Dabigatran-Clearance verlängert sein $[10,11]$. Dies sollte bei der Planung eines Eingriffs stets im Vorfeld berücksichtigt werden (Tab. 3).

Eine normale Thrombinzeit (mit einem thrombinreichen Kalibrator) schließt eine Wirkung von Pradaxa weitestgehend aus.

\section{Rivaroxaban $\left(\right.$ Xarelto $^{\circledR}$ )}

Der Hersteller empfiehlt ein Intervall von mindestens $18 \mathrm{~h}$ z.B. vor einer Epiduralkatheterentfernung und ein Intervall von $6 \mathrm{~h}$ vor der nächsten Einnahme, wobei dazu keine Studiendaten vorliegen. Abgeleitet von den pharmakologischen Kennzahlen kann bei therapeutischer Dosis eine Therapiepause von 22-26 h vor der Intervention angenommen und von 4-6 h nach der Intervention empfohlen werden. Bei nieren- und/oder leberfunktionseingeschränkten Patienten kann eine Kumulation eintreten. Daher ist es notwendig, eine zumindest qualitative, orientierende Aussage über die Wirkung bzw. restliche Wirkung von Rivaroxaban zu erhalten, wozu sich in aller Regel die Notfallbestimmung der TPZ («Quickwert») mit einem Rivaroxaban-empfindlichen Testkit (z.B. Neoplastin Plus) eignet. Ist eine deutliche Abweichung der TPZ vom Normbereich oder vom Vorwert des jeweiligen Patienten erkennbar, ist die Wirkung von Rivaroxaban mit großer Wahrscheinlichkeit gegeben [12]. Eine Messung des Anti-Faktor-Xa-Spiegels (kalibriert mit Rivaroxaban!) kann sinnvoll sein, um den Zeitpunkt der Operation individuell zu bestimmen. 
Tab. 4. Klinische Risikoabschätzung für thromboembolische Ereignisse unter OAK

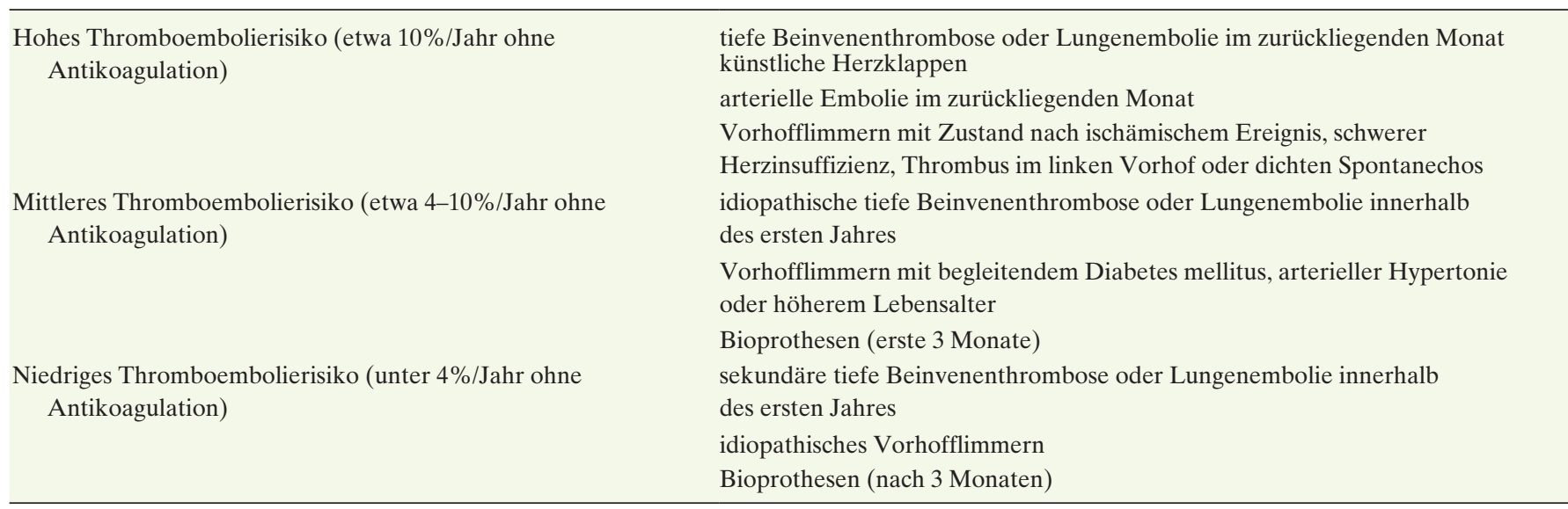

\section{Apixaban (Eliquis ${ }^{\circledR}$ )}

Bei prophylaktischer Dosis wird eine Therapiepause von 20-30 h vor der Intervention und von 4-6 h nach der Intervention empfohlen. Apixaban in therapeutischer Dosis sollte mindestens $48 \mathrm{~h}$ vor geplanten Operationen oder invasiven Eingriffen mit mittlerem bis hohem Blutungsrisiko abgesetzt werden. Bei nierenfunktionseingeschränkten Patienten kann eine Messung des Anti-Faktor-Xa-Spiegels (kalibriert mit Apixaban!) sinnvoll sein, um den Zeitpunkt der Operation individuell zu bestimmen [13].

\section{Heparinoide}

Aufgrund der alternativen Antikoagulation bei HIT-II und der Lieferschwierigkeiten ist zu erwarten, dass Danaparoid nur noch selten im klinischen Alltag eingesetzt werden wird. Bei geplanter Operation sollte auf eine präoperative Thromboseprophylaxedosis mit Heparinoid (Danaparoid) verzichtet werden. Aufgrund der langen Halbwertszeit sollte auch auf Katheterverfahren verzichtet werden. CAVE: Niereninsuffizienz.

\section{Orale Antikoagulanzien (Kumarine)}

Bei Pausierung wird ein Bridging mit NMH empfohlen. Der Zeitablauf des Bridgings richtet sich nach dem Zeitpunkt der Intervention/Operation und muss daher rechtzeitig - in der Regel mindestens 2 Wochen zuvor - mit dem Interventionalisten/Operateur abgesprochen werden. Dies wird - gerade durch die immer häufigere Verwendung von NMH - verstärkt zu einer Aufgabe der ambulanten Versorgung [8]. Auch postoperativ wird das Bridging heute häufig von niedergelassenen Ärzten beendet.

Das American College of Chest Physicians (ACCP) und die Deutsche Gesellschaft für Kardiologie (DGK) haben einen Leitfaden zum perioperativen Management der antithrombotischen Therapie vorgelegt [14, 15], der die Indikationen für ein Bridging auf der Basis des Blutungsrisikos nennt. Demnach kann auf ein Bridging verzichtet werden, wenn es sich bei dem bevorstehenden Eingriff um eine Zahnextraktion, eine kleine
Hautoperation oder Eingriffe im vorderen Augenabschnitt handelt. Solche «kleinen» Interventionen mit vergleichsweise geringem Blutungsrisiko können unter einer Antikoagulation mit INR-Werten um 2 durchgeführt werden. Praktisch bedeutet dies, dass die bisherige orale Antikoagulation (OAK) unverändert fortgesetzt oder - falls das Thromboembolierisiko des Patienten gering ist - kurz unterbrochen wird. Bei allen anderen größeren Eingriffen mit sehr hohem oder hohem Blutungsrisiko sollte nach aktuellem Wissensstand die OAK unterbrochen und ein Bridging durchgeführt werden (absolute Indikation). Allerdings sind die amerikanischen Empfehlungen dazu nicht immer eindeutig. Neben Art und Umfang der Operation spielt, wie bereits erwähnt, das individuelle Thromboembolierisiko des Patienten, das es abzuschätzen gilt, eine bedeutende Rolle bei der Entscheidung für oder gegen ein Bridging (Tab. 4).

Das periinterventionelle Vorgehen ist folgendermaßen (Abb. 2): Nach Absetzen des Vitamin-K-Antagonisten etwa 10 Tage vor dem geplanten Eingriff (tägliche INR-Kontrolle!) kommt es zu einem allmählichen Absinken der INR und des antikoagulatorischen Schutzes. Erreicht oder unterschreitet die INR bei den folgenden Messungen zum ersten Mal die untere Grenze des bisherigen Zielbereichs - meist 2,5 -, kann die NMHTherapie begonnen werden. Das NMH wird prä-, peri- und postoperativ appliziert, wobei sich die zu wählende NMH-Dosis am individuellen INR-Einstellungsbereich des Patienten orientiert. Eine Thrombozytenbestimmung am ersten Tag der NMHTherapie und dann mindestens zweimal pro Woche (wegen HIT-II) war bislang beim Bridging obligat, wird jedoch beim Einsatz von NMH nicht mehr gefordert $[15,16]$. Für NMH hat sich prä-, peri- und postoperativ eine halbtherapeutische Dosis von einmal täglich für Niedrigrisikopatienten und eine volltherapeutische Dosis in Form von halbtherapeutischer Dosis zweimal täglich für Hochrisikopatienten bewährt (Tab. 5). Auch Letztere ist gut verträglich und das Blutungsrisiko noch im tolerablen Bereich. Bei hohem Blutungsrisiko kann NMH am OP-Tag auch in Hochprophylaxedosis gegeben werden [15, 16]. Im Einzelfall kann auch ein Pausieren der NHM-Gabe am OP-Tag notwendig sein (Mitbetreuung von Spezialisten). 
Abb. 2. Präoperativer «Bridging»-Algorithmus bei OAK.

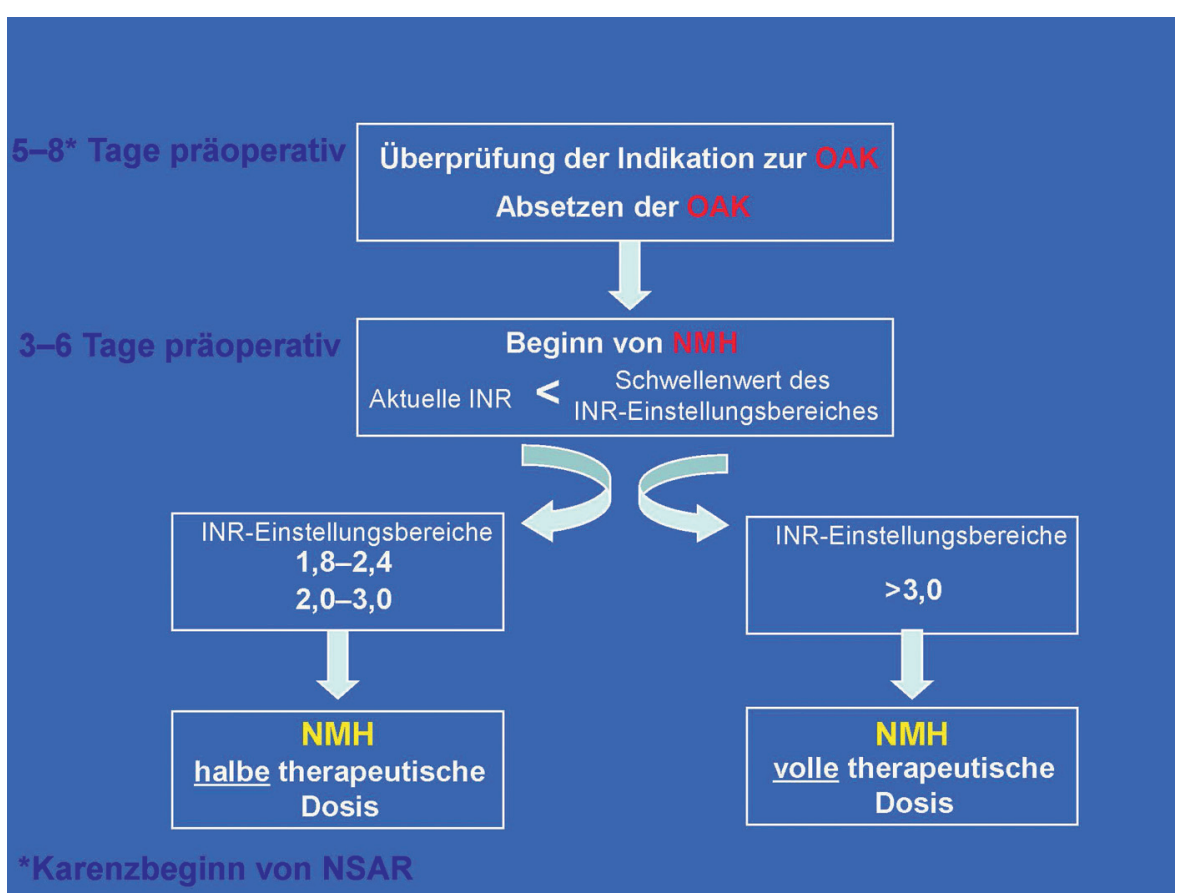

Tab. 5. Präinterventionelle Therapiepausen von gerinnungshemmenden Medikamenten in der zellulären Gerinnung (bei Patienten mit normaler Elimination und Organfunktion)

\begin{tabular}{|c|c|c|}
\hline $\begin{array}{l}\text { Gerinnungsmedikamente in der zellulären (thrombozytären) Gerinnung/ } \\
\text { Substanzen }\end{array}$ & $\begin{array}{l}\text { Therapieende + Labortestwert } \\
\text { frühestens vor Operation }\end{array}$ & $\begin{array}{l}\text { Therapiebeginn } \\
\text { nach Operation }\end{array}$ \\
\hline \multicolumn{3}{|l|}{ Adenosindiphosphat(ADP)-Rezeptorantagonisten } \\
\hline Clopidogrel & 7 Tage $^{a}$ & sofort $^{\mathrm{b}}$ \\
\hline Ticlopidin & 10 Tage $^{\mathrm{a}}$ & sofort $^{\mathrm{b}}$ \\
\hline Prasugrel & 7-10 Tage & $6 \mathrm{~h}$ \\
\hline Ticagrelor & 5 Tage & $6 \mathrm{~h}$ \\
\hline \multicolumn{3}{|l|}{ Cyclooxygenasehemmer } \\
\hline Acetylsalicylsäure & $100 \mathrm{mg}$ Monotherapie nicht erforderlich & sofort \\
\hline Nichtsteroidale Antirheumatika & nicht erforderlich & sofort \\
\hline Selektive COX-2-Hemmer & nicht erforderlich & sofort \\
\hline \multicolumn{3}{|l|}{ GP-IIb/IIIa-Inhibitoren } \\
\hline Abciximab & $48 \mathrm{~h}$ & $6 \mathrm{~h}$ \\
\hline Tirofiban & $8 \mathrm{~h}$ & $6 \mathrm{~h}$ \\
\hline Eptifibatid & $4 \mathrm{~h}$ & $6 \mathrm{~h}$ \\
\hline \multicolumn{3}{|l|}{ Antiaggregatorische Prostaglandine } \\
\hline Iloprost & $1 \mathrm{~h}$ & $6 \mathrm{~h}$ \\
\hline Prostacyclin (Epoprostenol) & $10 \mathrm{~min}$ & sofort \\
\hline Prostaglandin E1 & $10 \mathrm{~min}$ & sofort \\
\hline
\end{tabular}

Postoperativ wird die OAK wieder eingeleitet, wenn der Operateur das Blutungsrisiko postoperativ für vertretbar hält. Das ist meist am zweiten postoperativen Tag der Fall. Das Bridging mit NMH sollte so lange fortgesetzt werden, bis wieder ein sicherer Schutz durch die OAK gegeben ist. Das NMH wird erst dann abgesetzt, wenn die INR bei zwei aufeinanderfolgenden Messungen im Zielbereich liegt - meist nach 5-7 Tagen.

\section{Patienten mit gerinnungshemmender Therapie in der zellulären (thrombozytären) Gerinnung}

Alle beteiligten Ärzte (Operateur, Kardiologe, Hämostaseologe und Anästhesist) wägen das Risiko einer Stentthrombose gegen das einer operativ bedingten Blutung ab. Um besonders das Vorgehen bei Einzelfallentscheidungen zu erleichtern, sind den vorausgehenden Abschnitten die Faktoren 


\begin{tabular}{|c|c|c|c|}
\hline OP und. & Procedere & Ausnahme & $\begin{array}{l}\text { Procedere } \\
\text { mit Ausnahme }\end{array}$ \\
\hline $\begin{array}{c}\text { ASS } \\
\text { (Primärprophylaxe) }\end{array}$ & $\begin{array}{c}\text { STOPP von ASS, } \\
5 \text { d präinterventionell }\end{array}$ & & \\
\hline $\begin{array}{c}\text { ASS } \\
\text { (Sekundärprophylaxe) }\end{array}$ & $\begin{array}{l}\text { Fortführung } \\
\text { von ASS }\end{array}$ & $\begin{array}{l}\text { Hochrisikoeingriffe } \\
\text { (z.B. Neurochirurgie, } \\
\text { Körperhöhleneingriffe) }\end{array}$ & $\begin{array}{l}\text { STOPP von ASS, } \\
5 \text { d präinterventionell }\end{array}$ \\
\hline $\begin{array}{c}\text { ASS + Clopidogrel } \\
\text { in kardiovaskulären } \\
\text { Hochrisiko-Patienten } \\
\text { (z.B. Stent } \\
\text { DES < } 12 \text { (3) Monate } \\
\text { Kritische Stenosen) }\end{array}$ & $\begin{array}{l}\frac{\text { 1. Elektiveingriff: }}{\text { Verschieben bis }} \\
\text { kombinierte } \\
\text { PH nicht mehr } \\
\text { notwendig } \\
\frac{\text { 2. Semi-Elektiv: }}{\text { Fall zu Fall }} \\
\text { 3. Akut-OP: } \\
\text {-ASS/Clopidogrel weiter } \\
\text {-Antifibrinolytika, DDAVP } \\
\text { + TK in Bereitschaft }\end{array}$ & $\begin{array}{l}\text { Hochrisikoeingriffe } \\
\text { (z.B. Neurochirurgie, } \\
\text { Körperhöhleneingriffe) }\end{array}$ & $\begin{array}{c}\text { STOPP von } \\
\text { Clopidogrel } \\
5 \text { d präinterventionell, } \\
\text { dafür kurzwirkende } \\
\text { GPIIb/Illa-Antagonisten } \\
\text { STOPP von ASS } \\
\text { (Fall zu Fall-Entscheidung) }\end{array}$ \\
\hline
\end{tabular}

Abb. 3. Präoperativer Algorithmus unter Thrombozytenfunktionshemmern (nach Haemostasis in Critical Care (HICC) 2011 (www.hicc.ch)).
Blutungsrisiko und perioperatives Stentthromboserisiko gegenübergestellt. Bei einer elektiven Operation soll die Beendigung der Acetylsalicylsäure(ASS)/Clopidogrel-Medikation abgewartet werden. Bei dringlichen Operationen ist individuell zu entscheiden. Bei Akutoperationen ist eine Karenz der ASS/Clopidogrel-Medikation nicht möglich (Abb. 3).

Eine aktuelle Orientierung bieten die kardiologischen Empfehlungen des American College of Cardiology (ACC) mit weiteren Fachgesellschaften zur perioperativen Problematik einer ASS/Clopidogrel-Medikation bei Koronarstents $[17,18]$.

- Bei Patienten, bei denen in den nächsten 12 Monaten chirurgische Eingriffe zu erwarten sind, sollen unbeschichtete Stents (bare-metal stents (BMS)) implantiert werden.

- Elektive Eingriffe sollen bis zur Beendigung der Clopidogrel-Gabe verschoben werden, z.B. 3 Monate bei neuartigen medikamentenbeschichteten Stents (drug-eluting stents (DES)) oder bis 12 Monate bei DES.

- Wenn operative Eingriffe ein Absetzen einer ASS/Clopidogrel-Medikation erfordern, z.B. hohes Blutungsrisiko, soll - wenn möglich - zumindest ASS weiter gegeben werden.

- Das Problem lebensbedrohlicher Thromboembolien mit plötzlichem Herztod beim vorzeitigen Absetzen einer ASS/ Clopidogrel-Medikation bei Koronarstents muss den verantwortlichen Ärzten bewusst sein.

Bei elektiven Operationen sollte die Beendigung der plättchenhemmenden Kombinationsmedikation abgewartet werden. In den Leitlinien des ACC/der American Heart Association (AHA) wird empfohlen, elektive Operationen möglichst nach einem Zeitraum von 4 Wochen nach Implantation von BMS durchzuführen [17-19]. Da die Inzidenz später Stentthrombosen nach Implantation von DES aufgrund der verzögerten Endothelialisierung erhöht ist, ist ein längerer Zeitraum (bis zu 12 Monaten) nach Intervention mit hohem Risiko für perioperative Stentthrombosen zu erwarten. Somit sollte eine Mindestdauer einer ASS/Clopidogrel-Medikation von 6-12 Monaten nach Implantation von DES eingehalten werden und elektive Operationen sollten nach diesem Zeitraum durchgeführt werden. Umgekehrt sind bei früher geplanten Operationen eher BMS zu bevorzugen. Bei dringlichen Operationen und Patienten mit stabiler koronarer Herzerkrankung sollte eine perkutane transluminale koronare Angioplastie (PTCA) bzw. eine optimierte perioperative medikamentöse Therapie in Erwägung gezogen werden und gegebenenfalls eine Stentversorgung im ausreichenden postoperativen Intervall stattfinden [20].

Bei physiologischer Knochenmarksfunktion werden etwa 10\% der Thrombozyten pro Tag ersetzt, d.h., 4-5 Tage nach Absetzen von ASS weisen etwa 50\% der Thrombozyten eine normale Funktion auf.

Ist das Thromboserisiko hoch, können Clopidogrel und ASS möglichst bis zum Operationstag weiter gegeben werden. In den anderen Fällen kann die Clopidogrel-Medikation gestoppt und ASS als Monotherapie perioperativ weiter gegeben werden, wenn die aktuelle Blutungsanamnese negativ ist.

Eine perioperative Umstellung auf Heparine bei Patienten mit koronaren Stents bietet keinen ausreichenden Schutz vor Stentthrombosen. Standardheparin (UFH) führt sogar zu einer Plättchenaktivierung und NMH bietet keine ausreichende Plättchenhemmung [21]. Daher stellt die Gabe von Heparinen (Gerinnungshemmung) keine Alternative zu einer ASS/Clopidogrel-Medikation (Plättchenhemmung) dar.

Hier kann die ASS/Clopidogrel-Medikation perioperativ durch intravenös applizierbare Glykoprotein(GP)-IIb/IIIaAntagonisten mit kurzer Halbwertszeit, z.B. Tirofiban oder Eptifibatide, als alternative Plättchenhemmung ersetzt werden $[18,19]$. Tirofiban $\left(\right.$ Aggrastat $\left.^{\oplus}\right)$, ein synthetisches Nicht-Peptid, besitzt eine hohe GP-IIb/IIIa-Spezifität, eine Plasmahalbwertszeit von 90 min und eine Wirkdauer von 4-8 h. Eptifibatide $\left(\right.$ Integrilin $\left.^{\odot}\right)$, ein zyklisches Heptapeptid, weist bei einer Plasmahalbwertszeit von 150 min und einer Wirkdauer von 2-4 h ebenfalls eine hohe GP-IIb/IIIa-Spezifität auf. Abci- 
ximab (Reopro ${ }^{\odot}$ ) führt zu einer Plättchenhemmung bis $48 \mathrm{~h}$ nach Gabe - weit über seine Plasmahalbwertszeit hinaus - und scheidet als mögliche «Bridging»-Substanz aus. Bei nierenfunktionseingeschränkten Patienten ist der Einsatz von Eptifibatiden $\left(\right.$ Integrilin ${ }^{\odot}$ ) wegen der geringeren renalen Elimination $(<50 \%)$ gegenüber Tirofiban zu bevorzugen. Die intravenös zu applizierende Dosis von Tirofiban beträgt $0,1 \mu \mathrm{g} / \mathrm{kg} / \mathrm{min}$ aus einer kommerziellen Packungsgröße von $250 \mu \mathrm{g}$ pro $1 \mathrm{ml}$ Lösung. Eptifibatide sind mit $2 \mu \mathrm{g} / \mathrm{kg} / \mathrm{min}$, bei nierenfunktionseingeschränkten Patienten mit $1 \mu \mathrm{g} / \mathrm{kg} / \mathrm{min}$, aus einer kommerziellen Packungsgröße von $2 \mathrm{~g}$ pro $1 \mathrm{ml}$ Lösung intravenös über einen Perfusor zu verabreichen. Das heißt, dass für einen etwa 70-80 kg schweren Patienten eine Infusionsmenge von ungefähr $14 \mathrm{ml}$ Tirofiban pro $1 \mathrm{~h}$ und ungefähr $5 \mathrm{ml}$ Eptifibatiden pro $1 \mathrm{~h}$ bzw. ungefähr 2,5 ml Eptifibatiden pro $1 \mathrm{~h}$ bei nierenfunktionseingeschränkten Patienten benötigt wird.

In Tabelle 5 sind die wichtigsten Substanzen nochmals aufgeführt. Prasugrel $\left(\right.$ Efient $\left.^{\circledR}\right)$ ist ein Thienopyridin mit rascherem Wirkeintritt und $(10 \times)$ stärkerer, irreversibler Thrombozytenfunktionshemmung im Vergleich zu Clopidogrel. In der Phase-III-Studie war das Blutungsrisiko unter Prasugrel erhöht. Obwohl keine klinische Erfahrung vorliegt, sollte Prasugrel mindestens 7-10 Tage vor einer Blockade pausiert werden. Nach Intervention wird ein Intervall von $6 \mathrm{~h}$ empfohlen (von der ESA wird keine Pausezeit empfohlen).

Ticagrelor (Brilique ${ }^{\circledR}$ ) ist kein Thienopyridin und bindet (ohne Biotransformation) direkt an den P2Y12-Rezeptor. Auch Ticagrelor hat einen rascheren Wirkeintritt $(<2 \mathrm{~h})$ und eine stärkere und vorhersehbarere Thrombozytenfunktionshemmung im Vergleich zu Clopidogrel. Die Wirkungsdauer des reversiblen Thrombozytenhemmers beträgt 48-72 h. Obwohl keine klinische Erfahrung vorliegt, sollte Ticagrelor mindestens 5 Tage vor einer rückenmarksnahen Blockade pausiert werden. Nach Intervention wird ein Intervall von $6 \mathrm{~h}$ empfohlen. Das präoperative Prozedere in Abbildung 3 ist für Prasugrel und Ticagrelor übertragbar.

In Analogie zu ASS ist bei Monotherapie mit nichtsteroidalen Antirheumatika (NSAR) und unauffälliger Blutungsanamnese die Einhaltung von Therapiepausen vor rückenmarksnaher oder blutungsriskanter peripherer Regionalanästhesie nicht mehr zwingend empfohlen. Wenn ein gerinnungshemmender Effekt von NSAR mit Sicherheit ausgeschlossen werden soll, wird das Abwarten von 2 Halbwertszeiten empfohlen. Da zum Zeitpunkt einer Intervention zumeist eine Kombinationstherapie mit der medikamentösen Thromboseprophylaxe vorliegt, ist ein Zeitfenster von 2 Halbwertszeiten des NSAR als Pause empfehlenswert. Die Schmerztherapie sollte in dieser Phase mit Analgetika ohne Nebenwirkung der Thrombozytenfunktionshemmung erfolgen.

Da GP-IIb/IIIa-Inhibitoren beim akuten Koronarsyndrom in Kombination mit ASS und Antikoagulanzien eingesetzt werden und da folgende herzchirurgische Eingriffe Notfalloperationen darstellen, ist die Durchführung einer rückenmarksnahen Regionalanästhesie im Allgemeinen kontraindi- ziert. Bei der möglichen neuen Indikation von Tirofiban zum Bridging während der Clopidogrel-Pause sind aufgrund der kurzen Halbwertszeit (1,5 h) geringe Therapiepausezeiten empfohlen.

\section{Patienten mit hämorrhagischer Diathese}

Patienten mit vWS oder Thrombozytenfunktionsstörungen fallen perioperativ durch die Sofortblutung während und unmittelbar nach der Operation auf und können sich dadurch von Patienten mit plasmatischen Hämostasestörungen wie z.B. Hämophilie A und B oder anderen isolierten Faktorenmangelzuständen unterscheiden, die perioperativ in der Regel etwas später zu bluten beginnen. Zur Vermeidung dieser Blutungen sollten einige einfache perioperative Regeln grundsätzlich immer beachtet werden [22]:

- Diagnose und Festlegung des hämostaseologischen Managements, z.B. Substitutions- bzw. Medikamentenplan, vor der Operation;

- klinisches und hämostaseologisches Monitoring (die Laborwerte und der Patient müssen ärztlich zusammen begutachtet werden!);

- exakte zeitliche Einhaltung des hämostaseologischen Managements (unter anderem Laborkontrollen, Applikationszeitpunkte);

- ausreichende Depothaltung der benötigten blutstillenden Medikamente und Faktorenkonzentrate mit eindeutig geregelter Logistik (kurze Wege!);

- operativer Eingriff möglichst am Wochenanfang;

- möglichst keine oder sehr wenige invasive Methoden;

- Minimalisierung des Traumas bei der Operation;

- Vermeidung von intramuskulären (und intraarteriellen) Injektionen;

- bei unerwarteten Blutungen: zeitnahes hämostaseologisches Management.

Zusätzliche lokale Blutstillungsmaßnahmen wie sorgfältige blutungsverhütende Operationstechniken und der Gebrauch eines Fibrinklebers in einigen Operationsgebieten und -anwendungen (großen blutenden Parenchymflächen, blutenden gastrointestinalen Ulcera, zur Fixierung von Transplantaten und Implantaten wie Herniennetzen, zum Kleben von Nervenenden, zur Abdichtung von Gefäßprothesen, bei Nasenseptumplastiken und zur Abdichtung gegen Liquorleckagen) sind ebenfalls hilfreich zur Reduktion des potenziellen Blutungsrisikos [23]. Wegen der zusätzlichen Blutungsgefahr ist die Gabe von ASS (Dosierungen $\geq 600 \mathrm{mg}$ ) oder anderer NSAR als Schmerzmittel unmittelbar präoperativ kontraindiziert. Als alternative Analgetika für NSAR können Paracetamol, Cyclooxygenase-2(COX-2)-Hemmer, Tilidin-Naloxon oder Tramadol, die keine weiteren Hämostasestörungen verursachen, perioperativ eingesetzt werden $[4,5]$.

Als Nebenwirkungen der perioperativ applizierten Substitutionspräparate können selten allergische Reaktionen auf- 
Tab. 6. Perioperative Therapie hämorrhagischer Diathesen für Operationen mit hohem Blutungsrisiko

\begin{tabular}{|c|c|}
\hline Hämorrhagische Diathese & Therapeutisches Vorgehen (Präparat, Dosisberechnung, Zielwert) \\
\hline Fibrinogenmangel & $\begin{array}{l}\text { Fibrinogenkonzentrat } \\
\text { Fibrinogendosis }(\mathrm{g})=\text { erwünschter Anstieg }(\mathrm{g} / \mathrm{l}) \times \text { Plasmavolumen }(\mathrm{l}) \\
\text { Plasmavolumen }(\approx 40 \mathrm{ml} / \mathrm{kg} \text { Körpergewicht }) \\
\text { Folgedosis nach etwa } 36 \mathrm{~h} \\
>1 \mathrm{~g} / \mathrm{l}(0,5-1 \mathrm{~g} / \mathrm{l})^{\mathrm{a}}\end{array}$ \\
\hline Faktor-II-Mangel & $\begin{array}{l}\text { PPSB: } 1 \text { IE PPSB/kg Körpergewicht hebt die Aktivitäten der Faktoren VII und IX um 0,5-1\%, der Faktoren } \\
\text { II und X um } 1-2 \% \text { an! } \\
\text { Initialdosis (IE) = Körpergewicht }(\mathrm{kg}) \times \text { gewünschter Faktorenanstieg }(\%) \\
\text { maximal } 40 \text { (20) IE PPSB/kg Körpergewicht } \\
\text { Folgedosis nach etwa } 24 \mathrm{~h} \\
>50 \%(20-50 \%)\end{array}$ \\
\hline Faktor-V-Mangel & $\begin{array}{l}\text { Frischplasma } \\
15-20 \mathrm{ml} / \mathrm{kg} \text { Körpergewicht, Folgedosis nach etwa } 12 \mathrm{~h} \\
>20 \%\end{array}$ \\
\hline Faktor-VII-Mangel & $\begin{array}{l}\text { Faktor-VII-Konzentrat (plasmatisch) } \\
\text { Dosis IE = Körpergewicht }(\mathrm{kg}) \times \text { gewünschter Faktor-VII-Anstieg }(\%) \times 0,6 \text {, Folgedosis nach etwa } 3-5 \mathrm{~h} \\
\text { präoperativ }>50 \%(20-30 \%) \\
\text { dann } 25-45 \%(20-30 \%) \\
\text { oder } \\
\text { aktiviertes rFVIIa-Konzentrat } \\
15-30 \mu \mathrm{g} / \mathrm{kg} \text { Körpergewicht alle } 6 \mathrm{~h} \text { als Bolus für } 2 \text { Tage, dann im Verlauf alle } 8-12 \mathrm{~h} \\
\text { kein Zielwert } \\
\text { keine PPSB-Gabe (nur im Notfall)! }\end{array}$ \\
\hline Faktor-VIII/IX-Mangel & $\begin{array}{l}\text { Faktor-VIII/IX-Konzentrate (plasmatisch/rekombinant) } \\
\text { Initialdosis: Körpergewicht } \times 80-100(40-50)=\mathrm{IE}(\text { schwere Hämophilie }<1 \%) \\
\text { Initialdosis: Körpergewicht } \times 60-80(30-40)=\mathrm{IE}(\text { mittelschwere Hämophilie } 1-5 \%) \\
\text { Initialdosis: Körpergewicht } \times 50(25)=\mathrm{IE}(\text { leichte Hämophilie }>5-15 \%) \\
\text { Folgedosis: } 2-3 \text { täglich bei Hämophilie A, 1-2 täglich bei Hämophilie B (nach individueller Recovery) } \\
>50-100 \%(30-50 \%) \\
\text { keine PPSB-Gabe bei Faktor-IX-Mangel (nur im Notfall)! }\end{array}$ \\
\hline Faktor-X-Mangel & $\begin{array}{l}\text { PPSB: } 1 \text { IE PPSB/kg Körpergewicht hebt die Aktivitäten der Faktoren II und X um 1-2\% an! } \\
\text { Initialdosis (IE) = Körpergewicht }(\mathrm{kg}) \times \text { gewünschter Faktorenanstieg ( \%) } \\
\text { maximal } 40 \text { (20) IE PPSB/kg Körpergewicht } \\
\text { Folgedosis nach etwa } 24 \mathrm{~h} \\
>50 \%(20-50 \%)\end{array}$ \\
\hline Faktor-XI-Mangel & $\begin{array}{l}\text { Frischplasma (alternativ DDAVP) } \\
20 \mathrm{ml} / \mathrm{kg} \text { Körpergewicht, Folgedosis nach etwa } 24 \mathrm{~h} \\
>20 \%(15-20 \%)\end{array}$ \\
\hline Faktor-XIII-Mangel & $\begin{array}{l}\text { Faktor-XIII-Konzentrat } \\
\text { präoperativ bis zu } 35 \text { E/kg Körpergewicht Dosis, dann 10-20 E/kg Körpergewicht, Folgedosis meist nach etwa } 72 \text { h } \\
>50 \%\end{array}$ \\
\hline Prothrombinkomplex-Mangel & $\begin{array}{l}\text { PPSB: } 1 \text { IE PPSB/kg Körpergewicht hebt die Aktivitäten der Faktoren VII und IX um 0,5-1\%, der Faktoren } \\
\text { II und X um 1-2\% an! } \\
\text { Initialdosis (IE) = Körpergewicht }(\mathrm{kg}) \times \text { gewünschter Faktorenanstieg }(\%) \\
\text { maximal } 40 \text { (20) IE PPSB } / \mathrm{kg} \text { Körpergewicht } \\
\text { Folgedosis nach etwa } 6-8 \mathrm{~h} \\
\text { Quickwert: }>60 \%(30-50 \%)\end{array}$ \\
\hline vWF-Mangel & $\begin{array}{l}\text { Faktor-VIII-Konzentrate mit vWF }(1: 1) \text { (plasmatisch) } \\
\text { Initialdosis: Körpergewicht } \times 80(40-60)=\mathrm{IE} \\
\text { Folgedosis: } 1-2 \text { täglich (nach individueller Recovery) } \\
>50-100 \%(40-50 \%) \\
\text { oder DDAVP }\end{array}$ \\
\hline Hemmkörper (auch erworben) & $\begin{array}{l}\text { aktiviertes rFVIIa-Konzentrat } \\
\text { 90-120 } \mu \mathrm{g} / \mathrm{kg} \text { Körpergewicht alle } 2-3 \mathrm{~h} \text { als Bolus oder } 270 \mu \mathrm{g} / \mathrm{kg} \text { Körpergewicht alle } 8 \mathrm{~h} \text { für etwa } 2-3 \text { Tage, } \\
\text { dann im Verlauf alle } 6-8 \mathrm{~h} \\
\text { kein Zielwert } \\
\text { oder }\end{array}$ \\
\hline
\end{tabular}


Tab. 6. Fortsetzung

\begin{tabular}{|c|c|}
\hline Hämorrhagische Diathese & Therapeutisches Vorgehen (Präparat, Dosisberechnung, Zielwert) \\
\hline & $\begin{array}{l}\text { aktiviertes PPSB (aPPSB) } \\
100 \text { IE/kg Körpergewicht alle } 12 \mathrm{~h} \text { als Bolus für etwa 2-3 Tage, dann im Verlauf alle 12-24 h } \\
\text { kein Zielwert }\end{array}$ \\
\hline$\alpha-2$-Antiplasmin-Mangel & $\begin{array}{l}\text { Tranexamsäure } 1000 \mathrm{mg} \text { i.v. präoperativ, dann } 250 \mathrm{mg} / \mathrm{h} \text { i.v. intraoperativ bis } 4 \mathrm{~h} \text { nach OP-Ende } \\
\text { dann } 3 \times 500 \mathrm{mg} \text { p.o. bis } 3 \text { Tage postoperativ } \\
\text { kein Zielwert } \\
\text { oder } \\
\text { Antifibrinolytika, z.B. Aprotinin }{ }^{\mathrm{b}} \\
\text { Initialdosis: } 500000 \mathrm{KIE} \text { i.v. } \\
\text { Folgedosis: } 200000 \mathrm{KIE} / \mathrm{h} \text { i.v. über } 6-8 \mathrm{~h} \\
\text { kein Zielwert }\end{array}$ \\
\hline Thrombopenien & $\begin{array}{l}\text { wenn }<50000 / \mu l \\
\text { Thrombozytenkonzentrate } \\
\text { Thrombozytenkonzentrate ohne Effekt: Prednisolon-Gabe: } 100 \mathrm{mg} \text { präoperativ über } 4 \text { Tage } \\
\text { Prednisolon ohne Effekt: Immunglobulin-Gabe: } 0,4 \mathrm{~g} / \mathrm{kg} \text { Körpergewicht präoperativ über } 4 \text { Tage } \\
>80000 / \mu \mathrm{l} \text {; Zielwerte abhängig von Ursache der Thrombopenie und vom geplanten Eingriff } \\
\text { wenn } \geq 50000 \text { bis } 100000 / \mu \mathrm{l} \\
\text { DDAVP } \\
\text { Tranexamsäure } 500 \mathrm{mg} \text { i.v. präoperativ, dann } 250 \mathrm{mg} / \mathrm{h} \text { i.v. intraoperativ bis } 4 \mathrm{~h} \text { nach OP-Ende } \\
\text { dann } 3 \times 500 \mathrm{mg} \text { p.o. bis } 3 \text { Tage postoperativ } 90\end{array}$ \\
\hline $\begin{array}{l}\text { Thrombozytenfunktionsstörungen } \\
\text { (erworben, meist } \\
\text { medikamenteninduziert oder } \\
\text { organassoziiert) }\end{array}$ & $\begin{array}{l}\text { DDAVP } \\
\text { DDAVP ohne Effekt: Tranexamsäure } 1000 \mathrm{mg} \text { i.v. präoperativ, dann } 250 \mathrm{mg} / \mathrm{h} \text { i.v. intraoperativ bis } 4 \mathrm{~h} \text { nach } \\
\text { OP-Ende } \\
\text { dann } 3 \times 500 \mathrm{mg} \text { p.o. bis } 3 \text { Tage postoperativ } 90 \\
\text { Tranexamsäure ohne Effekt: Aprotinin }{ }^{\mathrm{b}} \\
\text { Initialdosis: } 500000 \mathrm{KIE} \text { i.v. } \\
\text { Folgedosis: } 200000 \mathrm{KIE} / \mathrm{h} \text { i.v. über } 6-8 \mathrm{~h} \\
\text { Antifibrinolytika ohne Effekt: konjugierte Östrogene } \\
\text { je } 25 \mathrm{mg} \text { i.v. präoperativ, intraoperativ und } 6-8 \text { h postoperativ } \\
\text { alle Medikamente ohne Effekt: Thrombozytenkonzentrate: } 1 \text { Apheresekonzentrat präoperativ, ein zweites } \\
\text { intraoperativ } \\
\text { Ziel: Normalisierung der jeweiligen pathologischen Thrombozytenfunktionstests }\end{array}$ \\
\hline $\begin{array}{l}\text { Thrombozytenfunktionsstörungen } \\
\text { (angeboren) }\end{array}$ & $\begin{array}{l}\text { DDAVP } \\
\text { DDAVP ohne Effekt: Tranexamsäure } 1000 \mathrm{mg} \text { i.v. präoperativ, dann } 250 \mathrm{mg} / \mathrm{h} \text { i.v. intraoperativ bis } 4 \mathrm{~h} \text { nach } \\
\text { OP-Ende } \\
\text { dann } 3 \times 500 \mathrm{mg} \text { p.o. bis } 3 \text { Tage postoperativ } 90 \\
\text { Tranexamsäure ohne Effekt: Aprotinin }{ }^{\mathrm{b}} \\
\text { Initialdosis: } 500000 \mathrm{KIE} \text { i.v. } \\
\text { Folgedosis: } 200000 \mathrm{KIE} / \mathrm{h} \text { i.v. über } 6-8 \mathrm{~h} \\
\text { Antifibrinolytika ohne Effekt: konjugierte Östrogene } \\
\text { je } 25 \mathrm{mg} \text { i.v. präoperativ, intraoperativ und } 6-8 \text { h postoperativ } \\
\text { alle Medikamente ohne Effekt und Thrombozytenkonzentrate nicht kontraindiziert: } \\
\text { Thrombozytenkonzentrate: } 1 \text { Apheresekonzentrat präoperativ, ein zweites intraoperativ } \\
\text { Thrombozytenkonzentrate ohne Effekt oder kontraindiziert: } 3 \text {-malige Bolusgabe rFVIIa }(80-120 \mu \mathrm{g} / \mathrm{kg} \\
\text { Körpergewicht) im Abstand von } 2 \mathrm{~h} \text { (z.B. Thromasthenie Glanzmann) } \\
\text { Ziel: Verbesserung oder sogar Normalisierung der jeweiligen pathologischen Thrombozytenfunktionstests }\end{array}$ \\
\hline $\begin{array}{l}\text { Hämorrhagische Diathese unklarer } \\
\text { Genese }\end{array}$ & $\begin{array}{l}\text { DDAVP } \\
\text { frisches Gefrierplasma (FFP): } 10 \mathrm{ml} / \mathrm{kg} \text { Körpergewicht } \\
\text { Thrombozytenkonzentrate: } 1 \text { Apheresekonzentrat präoperativ, ein zweites intraoperativ in Bereitschaft } \\
\text { Folgedosierungen nach klinischer Situation } \\
\text { keine Zielwerte }\end{array}$ \\
\hline
\end{tabular}

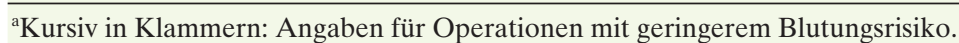

${ }^{\mathrm{b} D e r z e i t ~ k o m m e r z i e l l ~ n i c h t ~ v e r f u ̈ g b a r ! ~}$

treten, die durch Gabe von Antihistaminika und Kortikosteroiden beherrschbar sind. Die am meisten gefürchtete Komplikation sind Hemmkörper gegen intravenös zugeführte Faktoren (Faktor VII, VIII, IX, XI, XIII sowie den von-Wille-
brand-Faktor (vWF)). Diese treten im Erwachsenenalter äuBerst selten auf.

Das Auftreten von thromboembolischen Gefäßverschlüssen wird bei Patienten mit hämorrhagischen Diathesen selten 
beschrieben [24-28]. Daher wird eine medikamentöse Thromboseprophylaxe z.B. bei Patienten mit schwerer Hämophilie A und B oder Hemmkörperhämophilie in den meisten Zentren nicht durchgeführt. Grundsätzlich sollten alle Patienten mit einer hämorrhagischen Diathese unter perioperativer Substitutionstherapie eine physikalische Prophylaxe (Kompressionsstrümpfe, intermittierende pneumatische Kompression, Fuß-Impulstherapie, isometrische Übungen usw.) erhalten. Patienten mit hämorrhagischen Diathesen unter Substitutionstherapie sollten hinsichtlich der Plasmaspiegel von Gerinnungsfaktoren überwacht und dabei Spitzenspiegel deutlich über $100 \%$ vermieden werden. Eine Kombination mit NMH in prophylaktischer Dosis, z.B. für das hohe Risiko, sollte individuell in Abhängigkeit vom klinischen Gesamtbild in folgenden Situationen bedacht werden, wenn bei Operationen eine ausreichende Hämostase nach Substitution vorliegt:

- bei vorliegenden Komorbiditätsfaktoren (z.B. zusätzliche angeborene Hyperkoagulabilität durch Faktor-V-LeidenMutation, infektiöse/nichtinfektiöse Entzündungen und damit einhergehende Endothelzellaktivierung, große Traumata, Adipositas, Infektion mit dem humanen Immundefizienz-Virus (HIV), vorausgeganene Thromboembolie);

- bei möglichen thromboembolischen Risiken durch die Behandlung (z.B. Substitution von Faktorenpräparaten wie Faktor VII, VIII und besonders $I X$, ProthrombinkomplexKonzentrate (PPSB) sowie rekombinanter Faktor VIIa (rFVIIa) oder FEIBA ${ }^{\circledR}$; große operative Eingriffe mit hohem thromboembolischen Risiko, z.B. orthopädische Operationen, gelenkübergreifende Operationen);

- bei möglicher Gefährdung durch die klinische Situation (z.B. Langzeitvenenkatheter, Immobilisation).

Über die Dauer der einzelnen prophylaktischen Maßnahmen und den Wert einer kombinierten Anwendung bei Patienten mit unterschiedlichen hämorrhagischen Diathesen besteht derzeit kein Konsens. Für die Substitutionstherapie der meisten hämorrhagischen Diathesen hat die Bundesärztekammer bereits 2008 Leitlinien herausgegeben [23]. Die nachfolgend aufgeführten Dosierungen sind orientierend und können im Einzelfall individuell davon abweichen (Tab. 6).

Die Substitutionsdauer bzw. Anwendung der blutstillenden Medikamente erfolgt in der Regel bis zur Entfernung der Drainagen, der Nähte bzw. bis zur vollständigen Wundheilung. Diese ist abhängig von den jeweiligen Operationsverfahren.

Bei Operationen mit hohem Blutungsrisiko ist ein engmaschiges präoperatives Monitoring notwendig [23]. Die Häufigkeit der Laborkontrollen, Applikationskontrollen und der klinischen Kontrollen auf Blutungs- und Thrombosezeichen ist abhängig vom klinischen Schweregrad der hämorrhagischen Diathese und von den durchgeführten blutstillenden sowie operativen Maßnahmen. Präoperativ sollten die notwendigen Kontrollen am Vortag und morgens vor der Operation erfolgen. Der Operationsbeginn sollte genau eingehalten werden, z.B. 30-60 min nach Infusionsende einer Desmopressin(DDAVP)-Gabe oder einer Faktorengabe wie Faktor VIII, IX oder PPSB, da zu diesem Zeitpunkt in der Regel die Gipfelwerte der jeweiligen kritischen Faktoren erreicht werden. Am Operationstag (intraoperativ bei längeren Eingriffen und unmittelbar postoperativ sowie am Abend des Operationstages) sind bei den meisten hämorrhagischen Diathesen täglich zwei Kontrollen das Minimum. Bei perioperativen Blutungen wird ein umfangreicheres Monitoring notwendig, das individuell festgelegt wird.

Weitere Ursachen für perioperative Blutungen (unter anderem Hyperfibrinolyse, Hypothermie, Azidose, Massivtransfusion, Dilutionskoagulopathie) zusätzlich zu der bereits vorbestehenden hämorrhagischen Diathese dürfen nicht außer Acht gelassen werden. Selbst wenn keine Zielwerte vorgegeben sind, ist eine hämostaseologische Basisdiagnostik (aPTT, Quick, Fibrinogen, Thrombozytenzahl, Hämatokrit, Astrup mit pH, Kernkörpertemperatur und bei Verdacht auf Hyperfibrinolyse Thrombelastometrie) bei den Kontrollen obligat.

Bei komplikationslosem Verlauf können in der Regel ab dem zweiten postoperativen Tag die Kontrollen auf einmal täglich reduziert werden. Sofern Zielwerte für den Einsatz der blutstillenden Maßnahmen vorliegen, sollte das Erreichen dieser Zielwerte, z.B. des vWF:Ristocetin-Cofaktors (Rco), der meisten plasmatischen Gerinnungsfaktoren oder der Laborwerte von Thrombozytenfunktionstests, über die gesamte blutungsgefährdete Zeit angestrebt werden. Bei zahlreichen Thrombozytenfunktionsstörungen kann der PFA-100/PFA200 (Platelet Function Analyzer) zu einem Therapiemonitoring eingesetzt werden [29]. Überdosierungen der blutstillenden Maßnahmen sind zu vermeiden.

\section{Fazit}

Nicht elektive Situationen (Blutung bei Notoperationen, Akutpatienten) müssen oft ohne gezielte präoperative hämostaseologische Diagnostik und ohne Änderung der Gerinnungsmedikation rational therapiert werden. Elektive Eingriffe müssen sorgfältig geplant werden, besonders bei Patienten mit gerinnungshemmender Medikation oder hämorrhagischer Diathese.

\section{Disclosure Statement}

Frau Dr. Rutkauskaite hat keine Interessenkonflikte. Herr PD Dr. Koscielny ist/war Berater, Vortragender (Referent) und Studienleiter bzw. Prüfarzt für folgende Unternehmen: AstraZeneca, Baxter, Bayer, Biotest, Boehringer Ingelheim, CSL Behring, DRK, Ferring, GSK, Intersero, Leo, Octapharma, Novartis, Novo Nordisk, Pfizer, Sanofi Aventis und Siemens. 
1 Henseler O, Heiden M, Haschberger B, Hesse J, Seitz R: Bericht zur Meldung nach $\S 21$ TFG für die Jahre 2008 und 2009. Bundesgesundheitsbl 2010;53:1089-1103.

2 Koscielny J, Tempelhoff GF von, Ziemer S: A practical concept for preoperative management of patients with impaired primary hemostasis. Clin Appl Thromb Hemost 2004;10:155-166.

$>3$ Kessler P, Steinfeldt T, Gogarten W, Schwemmer U, Büttner J, Graf BM, Volk T: Peripheral regional anesthesia in patients under general anesthesia: risk assessment with respect to parasthesia, injection pain and nerve damage. Anaesthesist 2013. 62:483-488.

4 Pfanner G, Koscielny J, Pernerstorfer T, Gütl M, Perger P, Fries D, Hofmann N, Innerhofer P, Kneifl W, Neuner L, Schöchl H, Kozek-Langenecker SA: Präoperative Blutungsanamnese. Empfehlungen der Arbeitsgruppe Perioperative Gerinnung (AGPG) der Österreichischen Gesellschaft für Anästhesiologie und Intensivmedizin (ÖGARI). Anaesthesist 2007;56:604-611.

$\checkmark 5$ Koscielny J, Ziemer S, Radtke H, Pruss A, Schmutzler M, Sinha P, Salama A, Kiesewetter H, Latza R: A practical concept for preoperative identification in patients with impaired primary hemostasis. Clin Appl Thromb Hemost 2004;10:195-204.

6 Koscielny J, Ziemer S, Radtke H, Schmutzler M, Kiesewetter H, Salama A, von Tempelhoff GF: Preoperative identification of patients with impaired (primary) haemostasis. A practical concept. Hamostaseologie 2007;27:177-184.

7 Kozek-Langenecker SA, Fries D, Gütl M, Hofmann N, Innerhofer P, Kneifl W, Neuner L, Perger P, Pernerstorfer T, Pfanner G, Schöchl H: Empfehlungen der Lokoregionalanästhesien unter gerinnungshemmender Medikation. Empfehlungen der Arbeitsgruppe Perioperative Gerinnung (AGPG) der Österreichischen Gesellschaft für Anästhesiologie und Intensivmedizin (ÖGARI). Anaesthesist 2005;54:476-484.

8 Gogarten W, Vandermeulen E, Van Aken H Kozek S, Llau J, Samama M: Regional anaesthesia and antithrombotic agents: recommendations of the European Society of Anaesthesiology. Eur J Anaesth 2010;27:999-1015.

$\checkmark$ Rosencher N, Bonnet MP, Sessler DI: Selected new antithrombotic agents and neuraxial anaesthesia. Anaesthesia 2007;62:1154-1160.

-10 Spannagl M, Bauersachs R, Debus ES, Gawaz M, Gerlach H, Haas S, Hach-Wunderle V, LindhoffLast E, Riess H, Schellong S, Schinzel H, Bode C: Dabigatran therapy - perioperative management and interpretation of coagulation tests - Konsensus. Hamostaseologie 2012;32:294-305.
11 Food and Drug Administration (FDA): MiniSentinel Analyse Pradaxa ${ }^{\circledR}$ 2012. www.fda.gov/safety/ medwatch/safetyinformation/safetyalertsforhumanmedicalproducts/ucm282820.htm

12 Koscielny J, Beyer-Westendorf J, von Heymann C, Braun J, Klamroth R, Lindhoff-Last E, Tiede A, Spannagl M: Risk of bleeding and haemorrhagic complication with rivaroxaban - periprocedural management of haemostasis - Konsensus. Hamostaseologie 2012;32:287-293.

13 de Souza Brito F, Lopes RD, Alexander JH: The safety and efficacy of apixaban: where do we stand in 2013? Expert Opin Drug Saf 2013;12:559-567.

14 Douketis JD, Spyropoulos AC, Spencer A, Jaffer AK, Dunn AS; American College of Chest Physicians: Perioperative management of antithrombotic therapy: American College of Chest Physicians Evidence-Based Clinical Practice Guidelines, ed 9. Chest 2012;141(suppl 2):326-350.

15 Hoffmeister HM, Bode C, Darius H, Huber K, Rybak K, Silber S: Unterbrechung antithrombotischer Behandlung (Bridging) bei kardialen Erkrankungen. Positionspapier. Kardiologe 2010;4: 365-374.

16 Koscielny J, Ziemer S, von Heymann C: Patients with oral anticoagulation. Bridging anticoagulation in the perioperative phase. Hamostaseologie 2009; 29:247-255.

17 Grines CL, Bonow RO, Casey DE Jr, et al: Prevention of premature discontinuation of dual antiplatelet therapy in patients with coronary artery stents: a science advisory from the American Heart Association, American College of Cardiology, Society for Cardiovascular Angiography and Interventions, American College of Surgeons, and American Dental Association, with representation from the American College of Physicians. Circulation 2007;115:813-818.

18 Korte W, Cattaneo M, Chassot PG, Eichinger S, von Heymann C, Hofmann N, Rickli H, Spannagl M, Ziegler B, Verheugt F, Huber K: Peri-operative management of antiplatelet therapy in patients with coronary artery disease: joint position paper by members of the Working Group on Perioperative Haemostasis of the Society on Thrombosis and Haemostasis Research (GTH), the Working Group on Perioperative Coagulation of the Austrian Society for Anesthesiology, Resuscitation and Intensive Care (ÖGARI) and the Working Group Thrombosis of the European Society for Cardiology (ESC). Thromb Haemost 2011;105:743-749.
9 Koscielny J: Bleeding and thromboembolic risk. Perioperative strategy in aspirin/clopidogrel. Pharm Unserer Zeit 2009;38:352-358.

20 Carlsson J, von Wagenheim B, Linder R, Anwari TM, Qvist J, Petersson I, Magounakis T, Lagerqvist B: Is late stent thrombosis in drug-eluting stents a real clinical issue? A single-center experience and review of the literature. Clin Res Cardiol 2007;96:86-93.

21 Xiao Z, Theroux P: Platelet activation with unfractionated heparin at therapeutic concentrations and comparisons with a low-molecular-weight heparin and with a direct thrombin inhibitor. Circulation 1998;97:251-256.

22 Kußmann J, Koller M, Heinke T, Rothmund M: Die Bedeutung der präoperativen Gerinnungsanalytik für die Einschätzung des Blutungsrisikos in der Allgemeinchirurgie. Chirurg 1997;68:684-688.

23 Vorstand und Wissenschaftlicher Beirat der Bundesärztekammer (Hrsg): Querschnitts-Leitlinien (BÄK) zur Therapie mit Blutkomponenten und Plasmaderivaten, ed 4. Köln, Deutscher Ärzte-Verlag, 2008, pp 67-105.

24 Dargaud Y, Cruchaudet BB, Lienhart A, et al: Spontaneous proximal deep vein thrombosis in a patient with severe haemophilia A. Blood Coagul Fibrinolysis 2003;14:407-409.

25 Djulbegovic B, Hannan MM, Bergman GE: Concomitant treatment with factor-IX concentrates and antifibrinolytics in hemophilia-B. Acta Haematol 1995;94:43-48.

26 Franchini M, Krampera M, Veneri D: Deep vein thrombosis after orthopedic surgery in a patient with type I von Willebrand disease and mutations in the MTHFR and beta-fibrinogen genes. Thromb Haemost 2003;90:963-964

27 Ritchie B, Woodman RC, Poon MC: Deep venous thrombosis in hemophilia-A. Am J Med 1992;93: 699-700.

28 Small M, Jack AS, Lowe GDO, et al: Coronaryartery disease in severe hemophilia. Br Heart J 1983;49:604-607.

29 Koscielny J, Kiesewetter H, Tempelhoff GF: Platelet function analyzer (PFA)- $100^{\circledR}$ closure time in the evaluation of platelet disorders and platelet function. J Thromb Haemost 2006;4:1426-1427. 